03

\title{
Применимость приближенного уравнения Ланжевена для описания движения наносфер в поле стоячей световой волны
}

\author{
(C) А.А. Афранасьев ${ }^{1}$, Д.В. Новицкий ${ }^{1,29}$ \\ ${ }^{1}$ Институт фризики им. Б.И. Степанова НАН Беларуси, \\ 220072 Минск, Беларусь \\ ${ }^{2}$ Университет ИТМО, \\ 197101 Санкт-Петербург, Россия \\ ฯe-mail: dvnovitsky@gmail.com
}

Поступила в редакцию 24.05.2018 г.

На основе уравнения Ланжевена исследован процесс движения прозрачной наносферы в поле стоячей световой волны непрерывного лазерного излучения, а также определены условия ее локализации (оптической ловушки) в максимуме интерференционной картины поля двух встречных волн. Найдены границы применимости пренебрежения второй производной в уравнении Ланжевена - так называемого „укороченного уравнения“, использование которого позволяет получить решение в аналитическом виде. Определены условия устойчивости локализации наносферы в максимуме интерференционной картины поля в зависимости от кинетической энергии ее теплового (броуновского) движения.

DOI: $10.21883 /$ OS.2018.12.46937.146-18

\section{Введение}

Впервые перемещение и локализацию прозрачных частиц малых размеров силами светового давления излучения аргонового лазера реализовал А. Эшкин $[1,2]$, продемонстрировав эффект их левитации в воздухе. Им также наблюдалось горизонтальное перемещение частиц в воде вдоль направления распространения лазерного пучка. После его пионерских работ на стыке оптики, механики и лазерной физики сформировалось и стало успешно развиваться новое направление, изучающее процессы перемещения, манипуляции и локализации (оптические ловушки) частиц малых размеров силами светового давления, возникающими в поле лазерного излучения. Актуальность данного направления обусловлена прежде всего возможностью практического применения результатов в медико-биологических исследованиях (см. специальный выпуск [3]). Помимо медикобиологических применений силы светового давления могут быть использованы в качестве механизма концентрационной нелинейности в искусственно созданной гетерогенной среде - суспензии взвешенных прозрачных частицы в жидкости [4]. Такая суспензия, в которой каждая из компонент не проявляет нелинейно-оптических свойств, является высокоэффективной широкополосной нелинейной средой для непрерывного лазерного излучения сравнительно небольшой мощности [4,5]. Например, при исследовании четырехволнового смешения излучения аргонового лазера $\left(\lambda_{0}=5145 \AA\right)$ в водной суспензии латексных сферических частиц радиуса $R=1.17 \mu \mathrm{m} \mathrm{c}$ концентрацией $N_{0}=6.5 \cdot 10^{10} \mathrm{~cm}^{-3}$ авторы работы [4] измерили оптический коэффициент Керра, который ока- зался равным $n_{2}=3.6 \cdot 10^{-9} \mathrm{~cm}^{2} / \mathrm{W}$, что в $10^{5}$ раз больше, чем в сероуглероде $\mathrm{CS}_{2}$.

В работах [6,7] интерференционным полем излучения непрерывных лазеров осуществлена запись концентрационных решеток в водной суспензии полистирольных частиц с радиусами $R \approx 0.7-3 \mu \mathrm{m}$. Авторами работы [8] в сфокусированном гауссовом пучке $\mathrm{He}-\mathrm{Ne}$ лазера экспериментально реализована кристаллоподобная структура (двумерная сборка) вокруг центра пучка из сферических прозрачных частиц с $R \approx 3 \mu \mathrm{m}$. Интересный эксперимент по формированию двумерной матрицы полистирольных частиц с $R \approx 150-300 \mathrm{~nm}$ в воде под воздействием интерференционных пучков излучения двух непрерывных лазеров выполнен в [9] (рис. 1). Полученная двумерная матрица демонстрирует все характеристики обычных молекулярных кристаллов. При наличии слабого электрического поля авторы заметили увеличение размеров частиц, собираемых в матрицу. Эти результаты открывают перспективу синтезирования трехмерных матриц из прозрачных частиц малых размеров, оптическую сборку кристаллов-матриц, представляющих интерес для научных и практических приложений. Позже авторами работы [10] на основе решения уравнения Смолуховского теоретически предсказано формирование концентрационных паттернов из прозрачных наносфер (аналог двумерной сборки) в поле излучения встречных волн со случайной модуляцией их амплитуд (рис. 2). Все описанные выше результаты обусловлены действием градиентной составляющей силы светового давления.

В данной работе исследовано перемещение и локализация прозрачной наносферы градиентной компонентой силы светового давления в поле стоячей волны лазер- 


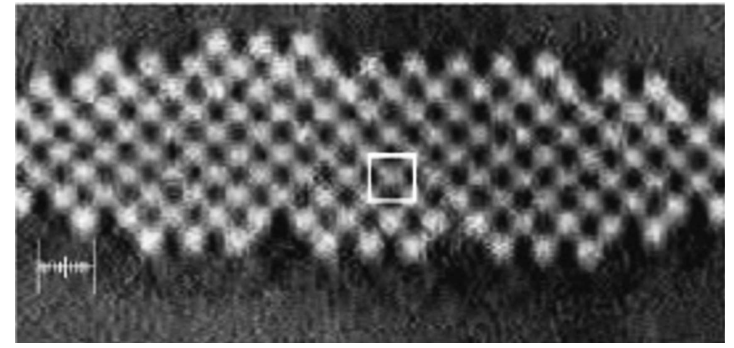

Рис. 1. Двумерная матрица полистирольных частиц, собранная градиентными силами в интерференционном поле двух лазеров [9].

ного излучения. Движение и локализация частиц малых размеров лазерным излучением является элементарным процессом формирования различных конфигураций из таких частиц, в частности решеток, кристаллоподобных структур, двумерных матриц и т. п.

\section{Основные уравнения и результаты}

В поле стоячей лазерной волны с интенсивностью $I(z)=I_{0}(1+\cos 2 k z)$ на прозрачную наносферу радиуса $R$ действует градиентная компонента силы светового давления, равная [11]

$$
\mathbf{F}_{\nabla}=-\mathbf{z} 4 \pi k \frac{n}{c} \alpha I_{0} \sin 2 k z
$$

где $\alpha=\frac{\bar{m}^{2}-1}{\bar{m}^{2}+2} R^{3}=\alpha_{0} R^{3}-$ поляризуемость наночастицы, $\bar{m}=n_{0} / n-$ отношение показателей преломления материала наносферы $n_{0}$ и окружающей ее жидкости $n$, $k-$ волновое число, $I_{0}=$ const. Движение наносферы под действием силы $\mathbf{F}_{\nabla}$ описывается уравнением Ланжевена [12]

$$
m \frac{d^{2} z}{d t^{2}}+6 \pi R \eta \frac{d z}{d t}=-F_{0} \sin 2 k z,
$$

где $z$ - координата центра наносферы с массой $m=4 \pi R^{3} \rho / 3$ и плотностью ее материала $\rho, 6 \pi R \eta-$ коэффициент трения в жидкости с вязкостью $\eta$, $F_{0}=4 \pi k n \alpha I_{0} / c$. При $\alpha_{0}>0$ уравнение (2) в безразмерной координате $x=2 k z$ удобно записать в виде

$$
\frac{d^{2} x}{d t^{2}}+\delta \frac{d x}{d t}+\Omega^{2} \sin x=0,
$$

где $\delta=9 \eta / 2 \rho R^{2}, \Omega^{2}=6 k^{2} n \alpha_{0} I_{0} / c$. В общем случае начальные условия уравнения (3) определяются соотношениями $x(t=0)=x$ и $(d x / d t)_{0}=2 k V_{0}$, где $V_{0}-$ начальная скорость наносферы.

При достаточно больших значениях коэффициента $\delta$ (например, для латексных наносфер с $\rho=1 \mathrm{~g} / \mathrm{cm}^{3}$ в воде при комнатной температуре $\eta=10^{-2} \mathrm{P}$ и $R \sim 10^{2} \mathrm{~nm}$ $\delta \approx 4.5 \cdot{ }^{8} \mathrm{~s}^{-1}$ ) вторая производная в (3) пренебрежимо мала, и можно использовать так называемое „укороченное уравнение“ (уравнение (3) при $d^{2} x / d t^{2}=0$ ) [13].
Для оценки применимости данного приближения используем среднее значение градиентной силы, заменив в (3) функцию $\sin x$ на ее среднее значение

$$
\langle\sin x\rangle=\frac{1}{\pi} \int_{0}^{\pi} \sin x d x=\frac{2}{\pi} .
$$

При этом решение уравнения (3) при $V_{0}=0$ можно записать в виде

$$
x(t)=x_{0}-2 k\left\langle V_{\nabla}\right\rangle t+\frac{2 k}{\delta}\langle V\rangle\left(1-e^{-\delta t}\right),
$$

где $\left\langle V_{\nabla}\right\rangle=\Omega^{2} / \pi k \delta-$ скорость наносферы под действием усредненной силы. В этом случае решение „укороченного уравнения“ (при $\left.d^{2} x / d t^{2}=0\right)$ определяется соотношением

$$
x(t)=x_{0}-2 k\left\langle V_{\nabla}\right\rangle t
$$

Легко видеть, что решения (4) и (5) будут практически совпадать при $\delta t \gg 1$. Время движения наносферы в точку $x=0$ (максимум интенсивности излучения) оценивается величиной $t \approx \pi / 2 k\left\langle V_{\nabla}\right\rangle$, и соответственно из условия $\delta t \gg 1$ находим

$$
\frac{1}{2} \frac{(\pi \delta)^{2}}{\Omega^{2}}=A \gg 1 .
$$

Таким образом, неравенство (6) является необходимым условием применимости „укороченного уравнения“ Ланжевена, решение которого имеет вид

$$
\operatorname{tg} \frac{x}{2}=\operatorname{tg} \frac{x_{0}}{2} e^{-V_{\nabla} t}
$$

где $V_{\nabla}=\Omega^{2} / \delta$.

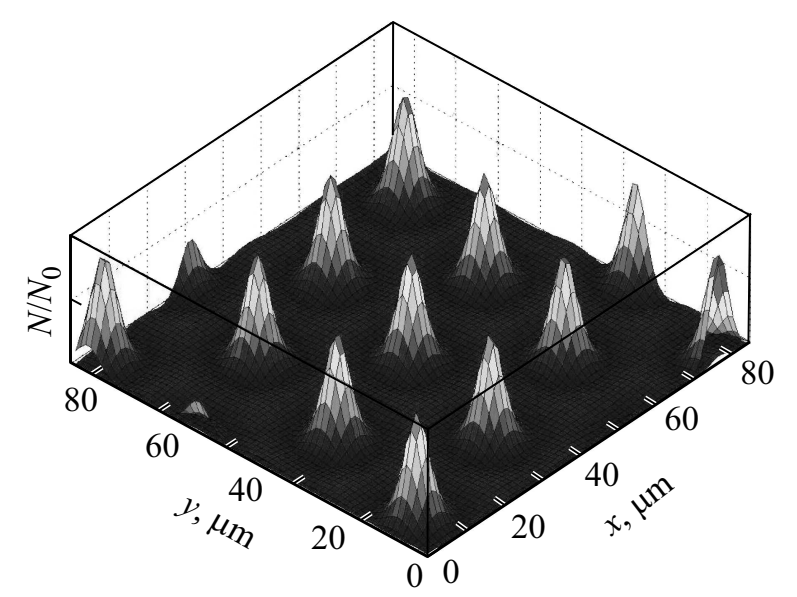

Рис. 2. Концентрационные паттерны прозрачных наносфер в поле стоячей лазерной волны со случайной модуляцией амплитуды. Результат численного моделирования уравнения Смолуховского [10], где $N_{0}-$ начальная концентрация наносфер, $(x, y)$ - плоскость, ортогональная оси распространения встречных волн. 

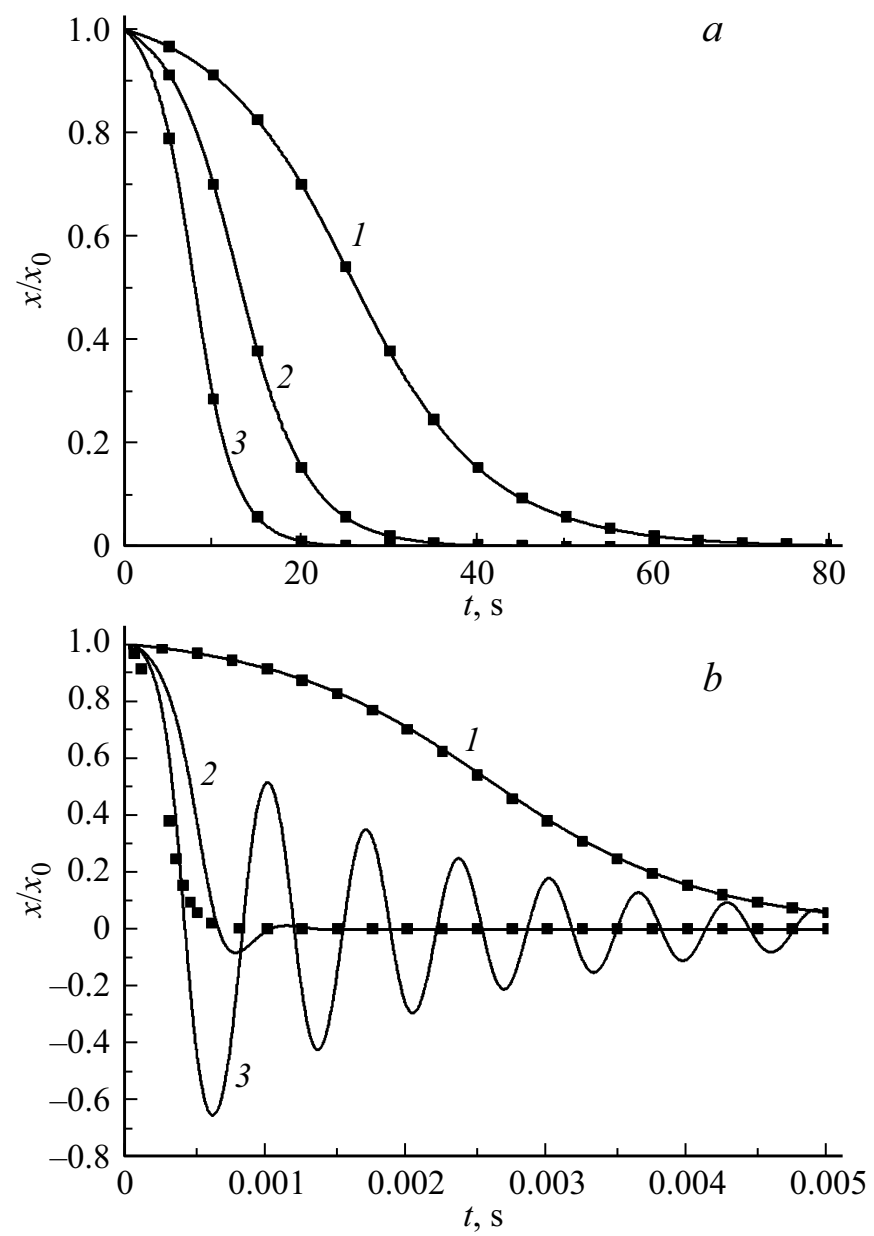

Рис. 3. Зависимость нормированной координаты центра наночастицы от времени при: (a) $\Omega^{2}=10^{9} ; \delta=10^{10}$ (1), $0.5 \cdot 10^{10}(2), 0.3 \cdot 10^{10}(3) ;(b) \Omega^{2}=10^{8} ; \delta=10^{5}(1), 10^{4}(2)$, $10^{3}$ (3). Точками показаны результаты решения укороченного уравнения.

В рис. 3 приведены временные зависимости координаты центра наносферы для различных значений коэффициентов $\Omega^{2}$ и $\delta$ при $x_{0}=0.95 \pi$. Сплошные кривые получены численным моделированием уравнения (3), а точки соответствуют аналитическому решению укороченного уравнения. Для всех кривых рис. 3, $a$ выполняется условие применимости укороченного уравнения $A \gg 1$, и соответственно решения уравнения (3) при $d^{2} x / d t^{2} \neq 0$ и $d^{2} x / d t^{2}=0$ полностью совпадают. На рис. $3, b$ условие $A \gg 1$ выполняется только для кривой 1 . Для кривой 2 условие применимости выполняется не строго $(A \approx 5)$, и соответственно решение укороченного уравнения незначительно отличается от решения уравнения (3). Это различие проявляется в основном в области малых $t$ и вблизи точки максимума интенсивности $x=0$, возле которой решение уравнения (3) проявляет тенденцию к колебательному движению. Для кривой $3 A \ll 1$, и соответственно укороченное уравнение не применимо. В этом случае наносфера совершает затухающие колебания около точки $x=0$.
Проведем оценку устойчивости локализации (двухпучковой ловушки) наносферы в максимуме интенсивности излучения. Как известно $[14,15]$, критерием устойчивости локализации является значительное превышение потенциала градиентной силы $F_{0}$ кинетической энергии теплового (броуновского) движения наносферы. В нашем случае этот критерий определяется соотношением

$$
2 \pi \frac{n}{c} \alpha_{0} R^{3} \frac{2 P}{\pi w_{0}^{2}} \geq 10 k_{B} T,
$$

где $k_{B}$ - постоянная Больцмана, $T$ - температура окружающей жидкости, $P$ и $w_{0}-$ соответственно мощность и радиус лазерных пучков, $I_{0}=2 P \cdot \pi w_{0}^{2}$.

Из (8) оценим минимальное значение радиуса $R_{\min }$ :

$$
R_{\min }=\left(\frac{2.5 k_{B} T c w_{0}^{2}}{n \alpha_{0} P}\right)^{1 / 3},
$$

при превышении которого наносфера устойчиво локализуется градиентной силой в максимуме интенсивности излучения. Например, для латексных наносфер в воде $(\bar{m}=1.65 / 1.33=1.24)$ при комнатной температуpe $\left(k_{B} T=4.14 \cdot 10^{-21} \mathrm{~J}\right)$ с использованием сфокусированных пучков аргонового лазера $\left(\lambda_{0}=5145 \AA\right)$ мощностью $P=100 \mathrm{~mW}$ диаметром $2 w_{0}=1.5 \lambda=0.58 \mu \mathrm{m}$ $\left(\lambda=\lambda_{0} / n\right)[14]$ из $(9)$ находим $R_{\min } \approx 23.5 \mathrm{~nm}$. Поскольку $R_{\min } \sim 1 / \sqrt{3} I_{0}$, то увеличением интенсивности излучения открывается возможность реализации двухволновой ловушки и для наносфер меньших размеров.

\section{Заключение}

На основании численного моделирования уравнения Ланжевена исследована динамика движения прозрачной наносферы в жидкости в поле стоячей лазерной волны под действием градиентной компоненты силы светового давления. Показано, что в определенной области параметров $\left(\Omega^{2}\right.$ и $\left.\delta\right)$ в используемом уравнении Ланжевена второй производной можно пренебречь, что позволяет получать результаты в аналитическом виде. Условием пренебрежения второй производной в (3) является неравенство $A=(\pi \delta)^{2} / 2 \Omega^{2} \gg 1$. Для случая $A \gg 1$ получены аналитические решения укороченного уравнения, полностью совпадающие с результатами численного моделирования исходного уравнения Ланжевена. Определен критерий устойчивости локализации наносферы (двухволновой оптической ловушки) в максимуме интерференционной картины излучения. Получена численная оценка минимального радиуса $R_{\min }$ наносферы, при превышении которого реализуется ее устойчивая локализация в максимуме интерференционной картины излучения. Для оценки значения $R_{\min }$ использованы экспериментальные значения параметров из работы [14].

Авторы благодарны академику А.Н. Рубинову за полезные обсуждения полученных результатов. Д.В.Н. признателен Российскому научному фонду (грант 17-72$10098)$ за поддержку в проведении численных расчетов. 


\section{Список литературы}

[1] Ashkin A. // Scientific American. 1972. V. 226. N 2. P. 63.

[2] Эикин А. // УФН. 1973. Т. 110. Вып. 1. С. 101.

[3] Спецвыпуск, посвященный использованию лазерных технологий в биофотонике и биомедицинских исследованиях // Квант. электрон. 2008. Т. 38. № 6; Special issue devoted to application of laser technologies in biophotonics and biomedical studies // Quantum Electron. 2008. V. 38. N 6.

[4] Smith P.W., Ashkin A., Tomlinson W.J. // Opt. Lett. 1981. V. 6. P. 284.

[5] Афанасьев А.А., Рубинов А.Н., Михневич С.Ю., Ермолаев И.Е. // ЖЭТФ. 2005. Т. 128. Вып. 3. C. 451; Afanas'ev A.A., Rubinov A.N., Mikhnevich S.Yu., Ermolaev I.E. // J. Exp. Theor. Phys. 2005. V. 101. N 3. P. 389.

[6] Burns M.M., Fournier J.M., Golovchenko J.A. // Phys. Rev. Lett. 1989. V. 63. N 12. P. 1233.

[7] Афанасьев А.А., Катаркевич В.М., Рубинов А.Н., Эфендиев T.Ш. // ЖПС. 2002. Т. 69. № 5. С. 675; Afanas'ev A.A., Katarkevich V.M., Rubinov A.N., Efendiev T.Sh. // J. Appl. Spectr. 2002. V. 69. N 5. P. 782.

[8] Rubinov A.N., Katarkevich V.M., Afanas'ev A.A., Efendiev T.Sh. // Opt. Commun. 2003. V. 224. P. 97.

[9] Mellor C.D., Bain C.D. // Chem. Phys. Chem. 2006. V. 7. N 2. P. 329.

[10] Afanas'ev A.A., Rubinov A.N., Volkov V.M., Izobov N.A., Mikhnevich S.Yu. // Nonl. Phen. Compl. Syst. 2008. V. 11. N 4. P. 465.

[11] Афанасьев А.А., Гайда Л.С., Гузатов Д.В., Рубинов А.Н., Свистун А.Ч. // Квант. электрон. 2015. Т. 45. № 10. С. 904; Afanas'ev A.A., Gaida L.S., Guzatov D.V., Rubinov A.N., Svistun A.Ch. // Quantum Electron. 2015. V. 45. N 10. P. 904.

[12] Абанасьев А.А., Гайда Л.С., Гузатов Д.В., Новицкий Д.В., Матук E.B. // Опт. спектр. 2016. Т. 120. № 1. С. 153; Afanas'ev A.A., Gaida L.S., Guzatov D.V., Novitski D.V., Matuk E.V. // Opt. Spectrosc. 2016. V. 120. N 1. P. 138.

[13] Афанасьев А.А., Новицкий Д.В. // Квант. электрон. 2017. T. 47. № 7. C. 651; Afanas'ev A.A., Novitsky D.V. // Quantum Electron. 2017. V. 47. N 7. P. 651.

[14] Ashkin A., Dziedzic J.M., Bjorkholm J.E., Chu S. // Opt. Lett. 1986. V. 11. P. 288.

[15] Harada Y., Asakura T. // Opt. Commun. 1996. V. 124. P. 529. 